\title{
Determinación de los parámetros de productividad de cepas de Pleurotus ostreatus y P. opuntiae cultivadas en paja de trigo y pencas de maguey combinadas con sustratos agrícolas
}

\author{
Determination of productivy parameters of $P$. ostreatus and $P$. opuntiae strains \\ cultivated on wheat straw and maguey leaves mixed with agricultural substrates
}

\begin{abstract}
Alejandro Portilla Segura ${ }^{1}$, Omar Romero-Arenas ${ }^{1}$, María de los Ángeles Valencia de lta ${ }^{1}$, Miguel Ángel Hernández Espinosa ${ }^{2}$, Gerardo Lanteta Cortés ${ }^{3}$, José Antonio Rivera-Tapia ${ }^{4}$
\end{abstract}

\footnotetext{
${ }^{1}$ Centro de Agroecología, Instituto de Ciencias, Benemérita Universidad Autónoma de Puebla (BUAP). Edificio VAL 1, Km 1,7 Carretera a San Baltazar Tetela, San Pedro Zacachimalpa, C.P. 72960, Puebla, México.

${ }^{2}$ Departamento de Investigación en Zeolitas y Posgrado en Agroecología, Instituto de Ciencias, BUAP. Puebla, México.

${ }^{3}$ Centro de Vinculación y Transferencia de Tecnología, BUAP. Prol. 24 Sury Av. San Claudio, Ciudad Universitaria, Col. San Manuel, C.P. 72570 , Puebla, México.

${ }^{4}$ Centro de Investigaciones en Ciencias Microbiológicas, ICUAP-BUAP. Edificio IC11, Ciudad Universitaria, C.P. 72570, Puebla, México.
}

\section{RESUMEN}

Antecedentes: La seta silvestre de maguey (Pleurotus opuntiae) es un alimento importante de las zonas semi-áridas del centro de México, apreciado por campesinos e indígenas desde tiempos antiguos, de sabor deseable y aprovechado en épocas de lluvias; sin embargo, hay pocos estudios acerca de su cultivo.

Objetivos: Comparar la producción y calidad nutritiva de basidiomas de $P$. opuntiae y $P$. ostreatus cultivados en paja de trigo, así como evaluar la combinación de diferentes residuos agrícolas combinados con penca de maguey para el cultivo de $P$. opuntiae, en condiciones controladas.

Métodos: Pleurotus opuntiae y $P$. ostreatus se cultivaron en pencas de maguey y residuos agrícolas pasteurizados a $80{ }^{\circ} \mathrm{C} / 2$ h. Se cuantificó la producción total, eficiencia biológica y tasa de producción. Se realizó un estudio químico proximal de los basidiomas obtenidos en los diferentes sustratos. El diseño estadístico fue por bloques al azar, además se realizó un análisis de componentes principales para determinar la correlación entre los rendimientos.

Resultados y conclusiones: Los mejores parámetros productivos se obtuvieron en paja de trigo con la cepa de P. ostreatus, pero $P$. opuntiae presentó un mayor contenido de proteína cruda y cenizas en la combinación de dos residuos agrícolas con penca de maguey.

Palabras clave: calidad nutricional, hongo de maguey, setas, sustratos agrícolas

\section{ABSTRACT}

Background: The wild maguey mushroom (Pleurotus opuntiae) is an important food of the semi-arid zones of the center of Mexico, appreciated by peasants and indigenous people since ancient times, of desirable flavor and used in rainy seasons; however, there are few studies about its cultivation.

Objectives: To compare the production and nutritional quality of $P$. opuntiae and $P$. ostreatus basidiomas grown in wheat straw, as well as to evaluate the combination of different agricultural residues combined with maguey leaves for the cultivation of $P$. opuntiae, under controlled conditions.

Methods: Pleurotus opuntiae and $P$. ostreatus were grown in maguey leaves and pasteurized agricultural residues at $80^{\circ} \mathrm{C} / 2 \mathrm{~h}$. Total production, biological efficiency and production rate were quantified. A proximal chemical study of the basidiomas obtained in the different substrates was performed. The statistical design was randomized, in addition an analysis of main components was performed to determine the correlation between the yields obtained.

Results and conclusions: The best productive parameters were obtained in wheat straw with the P. ostreatus strain, but $P$. opuntiae had a higher content of crude protein and ashes in the combination of two agricultural residues with maguey leaves.

Keywords: agricultural substrates, mushroom of maguey, nutritional quality, oyster mushroom

\section{ARTICLE HISTORY}

Received 18 July 2018 / Accepted 22 August 2019

Published on line: 12 October 2019

\section{CORRESPONDING AUTHOR}

José Antonio Rivera-Tapia, jart70@yahoo.com

ORCID: 0000-0002-2727-4584 


\section{INTRODUCCIÓN}

El género Pleurotus es un grupo de hongos comestibles que se cultiva en todo el mundo, ocupando el segundo lugar de producción (Sánchez, 2010; Bederska-Lojewska et al., 2017). Presenta un micelio con importante valor nutricional, debido a la presencia de altos contenidos de aminoácidos (arginina, alanina, glutamina, ácido glutámico). En $100 \mathrm{~g}$ de hongos frescos hay un $15 \%$ de la vitamina $\mathrm{C}$ recomendada como ingesta diaria para los seres humanos. Las especies de este género también contiene 0.1 a $0.2 \mathrm{~g}$ de grasas, incluyendo ácido oleico (40\%), ácido linoleico (55\%) y otros compuestos con acción hipocolesterolémica, características biológicas que lo hacen apropiado para su consumo y cultivo, así como de alto valor económico Barros et al. (2008).

El carpóforo desarrollado sobre diferentes sustratos lignocelulósicos (Carrasco-González et al., 2017), produce enzimas como las oxidorreductasas, celulasas, pectinasas, ligasas, proteasas y pectinasas (Rui-Heng et al., 2016), necesarias para la degradación de materia orgánica (Kurt y Buyukalaca, 2010). Además, contiene metabolitos bioactivos (polisacáridos, fenoles, terpenos, esteroles y sus derivados) que promueven atributos nutricionales y beneficios potenciales para la salud (Patel et al., 2012; Gomes et al., 2016).

El término setas es aplicado comercialmente en México para referirse a los hongos del género Pleurotus, entre ellos P. ostreatus, P. djamor, P. pulmonarius, y P. opuntiae; pero a nivel rural son conocidos como orejas blancas, orejas de palo, orejas de patancán, orejas de cazahuate u hongo del maguey (Das et al., 2010). Estas especies se producen de manera natural en materia orgánica en descomposición y pueden ser introducidos al cultivo utilizando diversos desechos agrícolas y agroindustriales, en ambientes controlados (Barros et al., 2008).

El hongo de maguey, P. opuntiae, es una especie crece en zonas semi-áridas del centro de México (Barrales y Mata, 2016) y es consumido por la población rural (Camacho et al., 2012). Es una de las setas silvestres consideradas como un manjar, consumidas desde tiempos antiguos por presentar un sabor y un aroma altamente deseable (Keles et al., 2011). Se le encuentra creciendo frecuentemente sobre especies de los géneros Opuntia, Phytolacca y Yucca en México, siempre asociado a plantas vivas y emergiendo de entre las hojas secas por la base de la planta (Barrales y Mata, 2016).
A pesar del amplio conocimiento que se tiene en México sobre especies comerciales de Pleurotus, la falta de recursos económicos y tecnológicos han limitado los estudios sobre el cultivo de hongos nativos del país, especialmente con cepas nativas del hongo de maguey, del cual existen pocos estudios (López et al., 2010; Barrales y Mata, 2016). El uso de bagazo de Agave salmiana y A. weberi o mezclas de bagazo de agave y hojas secas de maíz para la producción de $P$. ostreatus fue previamente reportado por Heredia-Solis (2014) y Chairez-Aquino et al. (2015) mientras que Barrales y Mata (2016) incursionaron en la producción de $P$. opuntiae en paja de cebada y hoja de caña, con resultados favorables.

Por lo anterior, el presente trabajo tuvo como objetivos: a) realizar una evaluación de la producción en sustrato de paja de trigo de una cepa silvestre de $P$. opuntiae, comparándolo con una cepa de $P$. ostreatus, b) evaluar las fructificaciones de $P$. opuntiae cultivadas en penca de maguey en combinación con diferentes residuos agrícolas $y, c)$ : realizar un análisis proximal de los basidiomas de $P$. opuntiae y $P$. ostreatus, con la finalidad de determinar la calidad de los cuerpos fructíferos obtenidos.

\section{MATERIALES Y MÉTODOS}

Esta investigación se llevó a cabo en la Planta Experimental de Producción de Setas Comestibles del Centro de Agroecología, en la Benemérita Universidad Autónoma de Puebla.

\section{Cepas estudiadas}

La cepa Ma-PO7 de Pleurotus opuntiae (Durieu \& Lév.) Sacc., está depositada en el Cepario de Hongos Comestibles del Centro de Agroecología y fue recolectada en el municipio de Tetela de Ocampo, Puebla, México. Fue identificada por regiones ITs y se encuentra depositada en el Gen Bank con numero de accesión MK757594, mientras que la cepa CP-50 de P. ostreatus (Jacq.) P. Kumm., proviene del Centro de Recursos Genéticos de Hongos Comestibles (CREGENHC) del Colegio de Postgraduados, campus Puebla. Ambas cepas se mantienen en medio de cultivo papa, dextrosa y agar (PDA) marca Bioxon (Sobal et al., 2007), a una temperatura ambiente de $25^{\circ} \mathrm{C}$ (Thongklang y Luangharn, 2016).

Residuos agrícolas empleados para la producción de basidiomas Se utilizaron los siguientes materiales lignocelulósicos agrícolas: paja de trigo (Triticum aestivum L.), rastrojo 
de maíz (Zea mays L.), pajilla de frijol (Phaseolus vulgaris L.) y penca de maguey seca (Agave salmiana Otto ex Salm.). Todos los materiales fueron obtenidos en la región de Tetela de Ocampo, Puebla, México.

\section{Siembra e inoculación de las cepas}

El inóculo se preparó con grano de trigo. El tratamiento consistió en hervir $25 \mathrm{~kg}$ de trigo durante 20 min en 50 $\mathrm{L}$ de agua y reposo durante $30 \mathrm{~min}$. Posteriormente se escurrió en dos recipientes de plástico con capacidad de $10 \mathrm{~kg}$ durante $60 \mathrm{~min}$; se le adicionó una proporción de 1 a $4 \mathrm{~g}$ de cal/yeso por cada $2 \mathrm{~kg}$ de grano húmedo. Se colocaron $500 \mathrm{~g}$ de granos de trigo preparados en frascos con capacidad de $700 \mathrm{~g}$ y esterilizados durante 60 min a $121{ }^{\circ} \mathrm{C}$. Cuando los frascos se enfriaron, se inocularon con $0.25 \mathrm{~cm}^{2}$ de agar con micelio de las cepas Ma-PO7 y CP-50, dentro de una campana de flujo laminar (VECCO, MÉXICO) y se incubaron a temperatura ambiente durante 30 días (Romero et al., 2010).

Para la siembra de las cepas, los sustratos agrícolas se trituraron mecánicamente en porciones de 1-2 cm de longitud con una picadora de forraje Modelo 1800 y fueron pasteurizados en agua caliente a $80{ }^{\circ} \mathrm{C}$ por $2 \mathrm{~h}$ (Bandopadhyay, 2013). Todos los tratamientos combinados se mezclaron manualmente antes de la pasteurización en una proporción de 1:1 y 1:1:1, manteniendo un $70 \%$ de humedad al finalizar la pasteurización (Thongklang y Luangharn, 2016). Transcurrido el tiempo de pasteurización, los sustratos se transportaron al área de siembra para permitir su enfriamiento y el escurrimiento del exceso de humedad (alrededor de 45 min). Posteriormente se procedió a la inoculación; se prepararon bolsas de plástico de 6 kg (peso húmedo) de cada tratamiento, sembradas homogéneamente con el inóculo previamente preparado en una relación 1:10 (Romero et al., 2010). Cabe mencionar que la cepa CP-50 de P. ostreatus sólo se inoculó en el sustrato de paja de trigo, considerándolo grupo control.

\section{Diseño experimental}

Se realizó el experimento con un diseño de bloques al azar, el cual contó con 9 tratamientos de $6 \mathrm{~kg}$ por unidad de producción (Tabla 2) con 10 repeticiones por tratamiento. Las bolsas sembradas se incubaron a temperatura ambiente $\left(26 \pm 2{ }^{\circ} \mathrm{C}\right)$. Cuando el micelio del hongo colonizó completamente los sustratos y se observó la aparición de primordios, las bolsas se tras- ladaron al cuarto de fructificación. Se mantuvieron a $27 \pm 1{ }^{\circ} \mathrm{C}, 70-80 \%$ de humedad, luz diurna indirecta y extracción de aire por 1 h, cada 8 h (Garzón y Cuervo, 2008; Romero et al., 2010). El riego se llevó a cabo cada mañana y tarde utilizando un rociador con agua del grifo (Andrino et al., 2011) durante el desarrollo y corte de los cuerpos fructíferos.

Se evaluó la eficiencia biológica ( $E B=$ peso de hongos frescos/peso de sustrato seco utilizado) x 100 (Salmones et al., 1997) y la tasa de producción ( $\mathrm{TP}=\mathrm{EB} /$ tiempo) transcurrido desde la inoculación hasta la última cosecha (García-Oduardo et al., 2011). La productividad (PT) se expresó en términos de gramos de hongos frescos por el número de cosechas totales (Romero et al., 2010; Chairez-Aquino et al., 2015).

Análisis químico proximal de los cuerpos fructíferos de $P$. opuntiae y P. ostreatus

El análisis químico proximal se realizó siguiendo los procedimientos recomendados por la American Association of Official Agricultural Chemists (AOAC, 1990; Feldsine et al., 2002). Todas las muestras de los cuerpos fructíferos obtenidos de los sustratos agrícolas de trigo, frijol, maíz, penca de maguey, así como sus respectivas combinaciones, fueron procesadas en el Laboratorio de Calidad Nutritiva de la Facultad de Medicina de Veterinaria de Tecamachalco, perteneciente a la Benemérita Universidad Autónoma de Puebla.

Los hongos se molieron hasta alcanzar un tamaño de partícula de 0.5 a $1 \mathrm{~cm}$. Se secaron en estufa a $55^{\circ} \mathrm{C}$ durante 48 h. A los residuos deshidratados, se les determinó proteína cruda (PC) con el método Micro-Kjeldahl (Carvalho et al., 2015), utilizando el factor de conversión de $\mathrm{N} \times 4.28$ (AOAC, 1990; 2004). La determinación de los lípidos fue llevada a cabo por la técnica de Golfish (AOAC No. 954.02, 1990). Las cenizas se determinaron a través de la incineración a $550{ }^{\circ} \mathrm{C}$ (AOAC No. 942.05, 1990) y la fibra cruda se determinó por el método Van Soest (2002). La materia seca de los materiales se obtuvo después de mantenerlos a $55^{\circ} \mathrm{C}$ durante $48 \mathrm{~h}$ (\% MS= [peso final de la muestra / peso inicial de muestra] $\times 100)($ Seibel, 1989; ARC, 1980). Los análisis se realizaron por triplicado para cada muestra obtenida de los diferentes tratamientos.

\section{Análisis estadístico}

Los valores obtenidos se procesaron en el programa estadístico SPSS Statistics versión 17 (Stadistical Package 
for the Social Sciences) para Windows, efectuando una prueba de $t$ de students para determinar diferencias significativas en la producción total, eficiencia biológica y tasa de producción de las cepas Ma-PO7 y CP-50, desarrolladas en sustrato de paja de trigo. Posterior al análisis de varianza (ANOVA) se aplicó la prueba de comparaciones múltiples de Tukey $(\alpha=0.05)$ para determinar las diferencias entre los 9 tratamientos. Además, se realizó un análisis de componentes principales con los datos de producción total obtenidos por las cepas.

\section{RESULTADOS Y DISCUSIÓN}

\section{Cuantificación de la PT, EB y TP de las cepas Ma-P07 y CP-50}

La producción total (PT) de la cepa CP-50 fue de 2 $271.66 \pm 0.44 \mathrm{~g}$, mientras que la cepa Ma-PO7 de $P$. opuntiae fue de $758.33 \pm 0.24 \mathrm{~g}$, registrando tres cosechas por tratamiento (Tabla 1). La cepa CP-50 de $P$. ostreatus produjo hongos de forma regular y de color blanco, mientras que la cepa Ma-PO7 de P. opuntiae produjo basidiocarpos de un color blanquecino. El mayor porcentaje de eficiencia biológica $(E B)$ se registró con la cepa CP-50, con $141.29 \pm 0.96 \%$ en paja de trigo (Tabla 1). En trabajos previos realizados con esta especie, Girmay et al. (2016) reportaron una EB de 74.17 \%, usando como sustrato semillas de algodón, mientras que Cayetano-Catarino et al. (2008) reportaron EBs de 99.8 y $96.4 \%$ para $P$. ostreatus y $P$. pulmonarius, cultivadas en una mezcla de tallos y hojas de plátano, valores menores a los alcanzados en el presente estudio. Por otra parte, con la cepa Ma-PO7 de P. opuntiae se obtuvo una EB de $59.22 \pm 0.27 \%$. Barrales y Mata (2016) mostraron EBs entre 86.15 y $105.21 \%$ con cepas silvestres de $P$. opuntiae en sustratos fermentados, datos superiores a los reportados en esta investigación. La mayor tasa de producción (TP) fue obtenida en con la cepa CP-50 de P. ostreatus con $2.04 \pm 0.003 \%$ (Tabla 1), donde se observa diferencias significativas con la prueba $t$ de student $(P \leq 0.05)$. El resultado fue bastante cercano al valor establecido por Romero et al. (2010) quienes reportaron una TP de $2.08 \%$ en paja de trigo. La cepa Ma-PO7 de P. opuntiae presentó $1.33 \pm 0.002 \%$ de TP en paja de trigo (Tabla 1), mientras que Barrales y Mata (2016) registraron una TP de $4.8 \%$ en paja fermentada inoculada con P. opuntiae. Cabe resaltar que el cálculo de la TP es importante desde el punto de vista comercial, ya que es recomendable disminuir los ciclos de cultivo para reducir los costos de producción (García-Oduardo et al., 2011).
TABLA 1. Parámetros de productividad de las cepas de Pleurotus opuntiae y $P$. ostreatus cultivadas en paja de trigo

\begin{tabular}{cccc}
\hline CEPA & $\begin{array}{c}\text { PRODUCCIÓN } \\
\text { TOTAL } \\
(\mathrm{G})^{*}\end{array}$ & $\begin{array}{c}\text { EFICIENCIA } \\
\text { BIOLÓGICA } \\
(\%)^{*}\end{array}$ & $\begin{array}{c}\text { TASA DE } \\
\text { PRODUCCIÓN } \\
(\%)^{*}\end{array}$ \\
\hline CP-50 & $2271.66 \pm 0.44^{\mathrm{a}}$ & $141.29 \pm 0.96^{\mathrm{a}}$ & $2.04 \pm 0.003^{\mathrm{a}}$ \\
Ma-PO7 & $758.33 \pm 0.24^{\mathrm{b}}$ & $59.22 \pm 0.27^{\mathrm{b}}$ & $1.33 \pm 0.002^{\mathrm{b}}$ \\
$\mathrm{F}_{\text {cal. }}$ & 1.667 & 5.341 & 0.488 \\
$\mathrm{P}$ & 0.001 & 0.043 & 0.001 \\
\hline
\end{tabular}

${ }^{*}$ Medias con letras diferentes en la misma columna indican diferencias significativas con la prueba de $t$ de student $(P \leq 0.05) . F_{\text {cal }}$ Valor calculado. P: Significancia.

Cuantificación de la PT, EB y TP de la cepa Ma-P07 utilizando sustratos agrícolas mezclados con penca de maguey La combinación de dos o más residuos agrícolas incrementó la producción de la cepa Ma-PO7, ya que, al mezclarlos con la penca de maguey, se logró aumentar la producción de los basidiocarpos en fresco, EB y la TP. El mayor porcentaje de EB y TP fue para el tratamiento $\mathrm{Mg}+\operatorname{Tr}+\mathrm{Fr}$ (Tabla 2) con $89.81 \pm 0.88 \%$, de EB y $1.71 \pm 0.04$ de TP, resultados similares a los reportados por Barrales y Mata (2016), con cepas silvestres de $P$. opuntiae.

TABLA 2. Eficiencias biológicas y tasas de producción alcanzadas por $P$. opuntiae (Ma-P07) en los diferentes tratamientos evaluados

\begin{tabular}{|c|c|c|c|}
\hline Código & DESCRIPCIÓN & $\begin{array}{c}\text { EFICIENCIA } \\
\text { BIOLÓGICA (\%) }\end{array}$ & $\begin{array}{c}\text { TASA DE PRODUCCIÓN } \\
(\%)\end{array}$ \\
\hline $\mathrm{Fr}$ & Paja de frijol & $47.92 \pm 0.28^{h}$ & $1.34 \pm 0.04^{b}$ \\
\hline $\mathrm{Ma}$ & Rastrojo de maíz & $59.61 \pm 0.96^{f}$ & $1.19 \pm 0.01^{\mathrm{bcd}}$ \\
\hline $\mathrm{Mg}$ & Penca de maguey & $74.41 \pm 0.50^{c}$ & $1.19 \pm 0.02^{\mathrm{bcd}}$ \\
\hline $\mathrm{Mg}+\mathrm{Tr}$ & $\begin{array}{c}\text { Penca de maguey }+ \\
\text { paja de trigo }(1: 1)\end{array}$ & $69.59 \pm 0.83^{d}$ & $1.22 \pm 0.05^{\mathrm{bcd}}$ \\
\hline $\mathrm{Mg}+\mathrm{Ma}$ & $\begin{array}{l}\text { Penca de maguey + } \\
\text { rastrojo de maíz (1:1) }\end{array}$ & $63.68 \pm 0.20^{e}$ & $1.65 \pm 0.05^{\mathrm{a}}$ \\
\hline $\mathrm{Mg}+\mathrm{Fr}$ & $\begin{array}{c}\text { Penca de maguey + } \\
\text { paja de frijol }(1: 1)\end{array}$ & $57.89 \pm 0.78^{f}$ & $1.11 \pm 0.08^{\mathrm{de}}$ \\
\hline $\begin{array}{c}\mathrm{Mg}+\mathrm{Tr}+- \\
\mathrm{Fr}\end{array}$ & $\begin{array}{c}\text { Penca de maguey }+ \\
\text { paja de trigo + paja de } \\
\text { frijol }(1: 1: 1)\end{array}$ & $89.81 \pm 0.88^{a}$ & $1.71 \pm 0.04^{a}$ \\
\hline $\begin{array}{c}\mathrm{Mg}+- \\
\mathrm{Ma}+\mathrm{Tr}\end{array}$ & $\begin{array}{l}\text { Penca de maguey + } \\
\text { rastrojo de maíz + paja } \\
\text { de trigo (1:1:1) }\end{array}$ & $80.01 \pm 0.08^{b}$ & $1.26 \pm 0.01 b c$ \\
\hline $\begin{array}{l}\mathrm{Mg}+- \\
\mathrm{Ma}+\mathrm{Fr}\end{array}$ & $\begin{array}{l}\text { Penca de maguey + } \\
\text { rastrojo de maíz + paja } \\
\text { de frijol (1:1:1) }\end{array}$ & $71.20 \pm 0.81^{d}$ & $1.32 \pm 0.05^{\mathrm{bc}}$ \\
\hline
\end{tabular}

*Medias con letras diferentes en las columnas indican diferencias significativas con la prueba de Tukey $(P \leq 0.05)$. 


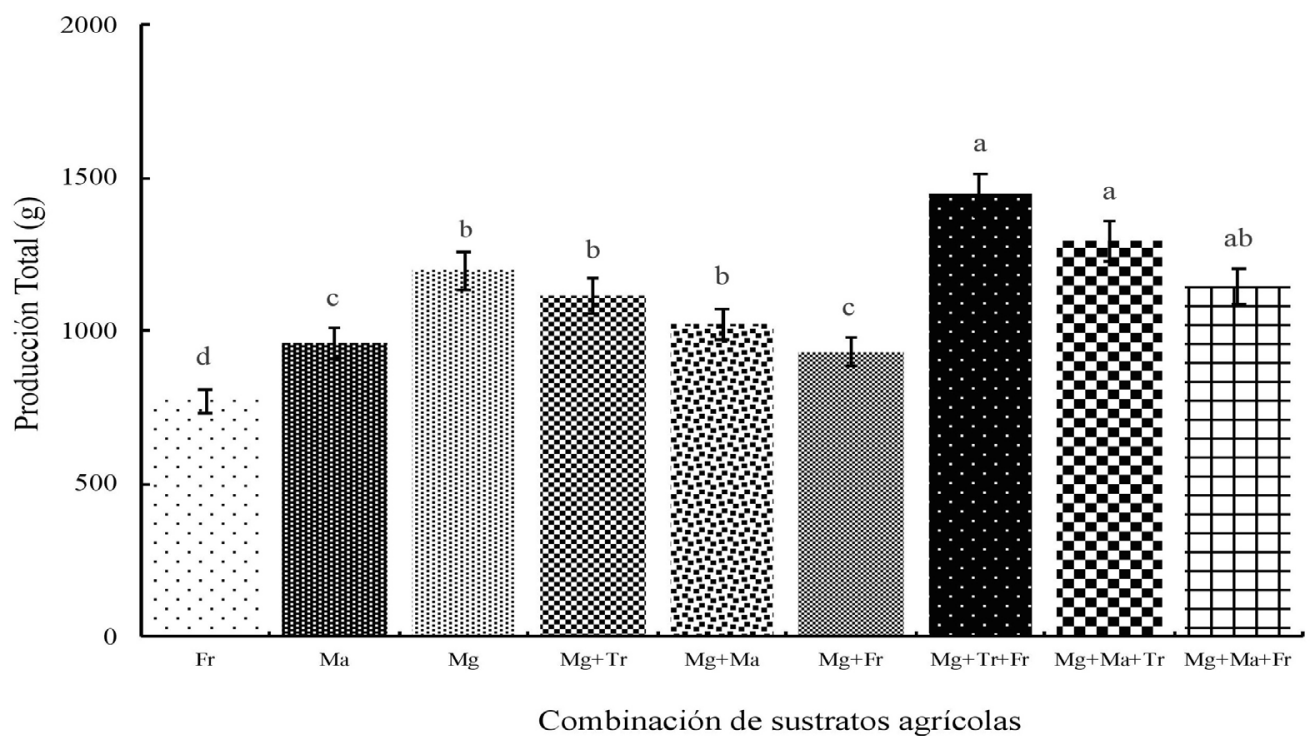

FIgURA 1. Producción total en peso fresco de la cepa Ma-PO7 de Pleurotus opuntiae en diferentes sustratos agrícolas combinados con penca de maguey. $\mathrm{Fr}=$ Paja de frijol; $\mathrm{Ma}=$ Rastrojo de maíz; $\mathrm{Mg}=$ Penca de maguey; $\mathrm{Mg}+\mathrm{Tr}=$ Penca de maguey + paja de trigo (1:1); $\mathrm{Mg}+\mathrm{Ma}=$ Penca de maguey + rastrojo de maíz (1:1); Mg+F= Penca de maguey + paja de frijol (1:1); Mg+Tr+Fr= Penca de maguey + paja de trigo + paja de frijol (1:1:1); Mg+Ma+Tr= Penca de maguey + rastrojo de maíz + paja de trigo (1:1:1); Mg+Ma+Fr= Penca de maguey + rastrojo de maíz + paja de frijol (1:1:1). *Medias con letras diferentes indican diferencias significativas con la prueba de Tukey $(\mathrm{P} \leq 0.05)$.

La mayor producción total (PT) de la cepa Ma-PO7 de $P$. opuntiae fue de $1446 \pm 0.16 \mathrm{~g}$, registrando en la combinación Mg+Tr+Fr (Figura 1), presentando diferencias significativas $(P=003)$ con los demás tratamientos. La menor PT se observó en el sustrato de pajilla de frijol, con $771 \pm 0.22 \mathrm{~g}$. Barrales y Mata (2016) registraron una producción de $345.1 \mathrm{~g}$ en paja de cebada y hoja de caña fermentadas, inoculada con la cepa IE 837 de P. opuntiae, resultados menores a los presentados en esta investigación. Cabe resaltar que la cepa estudiada por estos autores proviene del estado de Puebla, al igual que la cepa Ma-PO7.

El análisis de componentes principales mostró que la variable PT está altamente correlacionada para los diferentes rendimientos, ya que explica mejor la varianza (Figura 2). El $80.079 \%$ de la varianza fue explicada por PC1 con el $62.036 \%$ y por PC2 representando el $18.043 \%$, el PC1 se correlacionó negativamente con el rasgo del rendimiento más bajo, sin embargo, el PC2 se correlacionó con el mayor rendimiento obtenido con la cepa CP-50 de P. ostreatus. Los grupos de variables que denotan alta correlación positiva entre ellas son los rendimientos obtenidos con dos sustratos agrícolas más la penca de maguey, seguidos por la combinación de penca de maguey y un residuo agrícola; que están dentro del intervalo de 961 a 1446 g, además CP1 se correlacionó negativamente con los rendimientos menores a $800 \mathrm{~g}$ en peso fresco, que se presentaron en los tratamientos con paja de trigo y frijol, con la cepa Ma-PO7 de P. opuntiae.

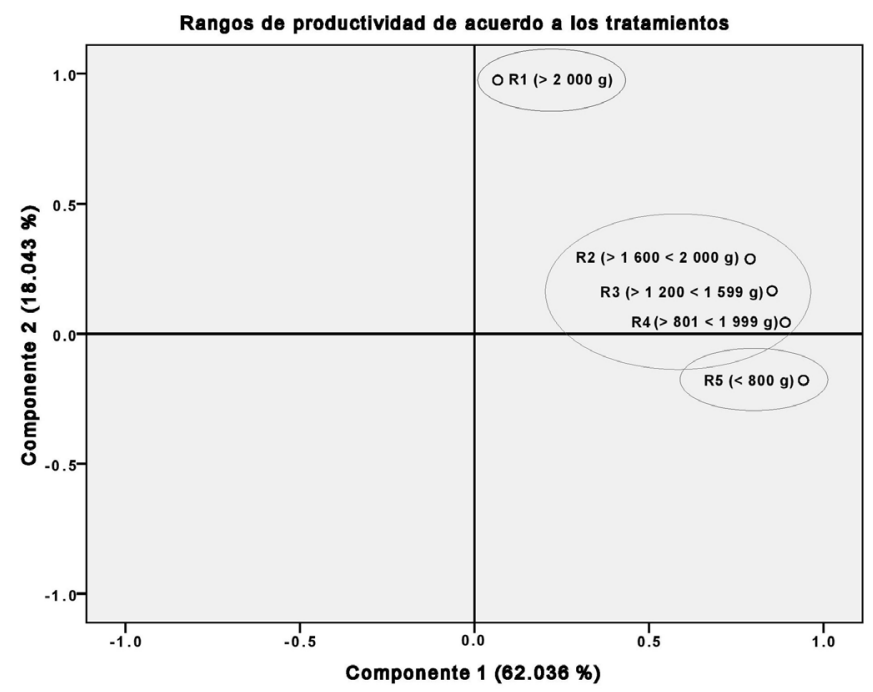

Figura 2. Análisis de componentes principales entre los rendimientos obtenidos en las diferentes combinaciones de sustratos utilizados para el cultivo de las de cepas de Pleurotus opuntiae y $P$. ostreatus. R1= Rendimiento mayor a 2, 000 g; R2= Rendimiento mayor a 1,600 g y menor a 2, $000 \mathrm{~g} \mathrm{R}=$ Rendimiento mayor a $1,200 \mathrm{~g}$ y menor a 1, $599 \mathrm{~g}$; R4= Rendimiento mayor a $801 \mathrm{~g}$ y menor a 1, $999 \mathrm{~g}$; R5= Rendimiento menor a $800 \mathrm{~g}$. 
Análisis químico proximal de basidiocarpos de la cepa CP-50 y Ma-P07 obtenidos de diferentes sustratos

Se determinó una gran similitud entre la composición de P. opuntiae cultivado en diferentes sustratos en diversas combinaciones, así como para P. ostreatus desarrollado en paja de trigo. La diferencia más notable entre ambas especies fue el contenido total de cenizas, fibra cruda y porcentaje extracto etéreo (Tabla 3). Los sustratos combinados con penca de maguey en diversas concentraciones presentaron una mayor concentración en proteína cruda, teniendo diferencias significativas con la prueba de Tukey ( $P \leq 0.05)$. Los resultados de porcentaje de proteína cruda (PC) en los cuerpos fructíferos de la cepa Ma-PO7 osciló entre 21.56 y $37.78 \pm 0.36$, siendo la combinación de paja de frijol con rastrojo de maíz y penca de maguey la más alta (37.78\%), mientras que para los basidiomas de la CP-50 (grupo control) se obtuvo un $26.75 \%$. Estu- dios realizados por Tolera y Abera (2017) presentaron $28.85 \%$ de PC en cuerpos fructíferos de P. ostreatus crecidos en paja de trigo, mientras que Yamauchi et al. (2018) informaron un $43.8 \%$ de PC en hongos setas producidos en aserrín de bambú fermentado mezclado con semillas de batata, resultados superiores a los encontrados en la presente investigación. Las variaciones en los contenidos de proteína en los cuerpos fructíferos de $P$. opuntiae y $P$. ostreatus cultivados en los diferentes tratamientos podría deberse al contenido variable de nitrógeno de los sustratos, como lo indican Hoa et al. (2015) y Michael et al. (2011). El contenido de humedad $(\mathrm{H})$ en los basidiomas de $P$. opuntiae osciló entre 89.82 a $93.37 \%$, con un promedio general de $91.19 \%$. Çağlarırmak (2007) obtuvo el $92.63 \%$ de contenido de $\mathrm{H}$ en $\mathrm{P}$. ostreatus, resultados similares a los obtenidos en los basidiomas de la CP-50 de P. ostreatus con $93.28 \%$ de H. La materia seca (MS) en los cuerpos fruc-

TABLA 3. Composición proximal de los cuerpos fructíferos de $P$. opuntiae y $P$. ostreatus cultivados en diferentes sustratos agrícolas

\begin{tabular}{|c|c|c|c|c|c|c|c|c|c|}
\hline \multirow{2}{*}{ Cepas } & \multirow{2}{*}{ Código } & PC & $\mathrm{H}$ & MS & $\mathrm{C}_{\mathrm{E}}$ & MO & $\mathrm{FC}$ & $\mathrm{EE}$ & $\mathrm{PH}$ \\
\hline & & \multicolumn{8}{|c|}{$\%$} \\
\hline \multirow[t]{5}{*}{ CP-50 } & $\operatorname{Tr}$ & $26.75 \pm 0.25^{\mathrm{e}}$ & $93.28 \pm 0.07^{a}$ & $6.72 \pm 0.04^{c}$ & $2.45 \pm 0.12^{9}$ & $97.55 \pm 0.12^{\mathrm{a}}$ & $14.67 \pm 0.80^{\mathrm{a}}$ & $1.95 \pm 0.02^{c}$ & $6.13 \pm 0.02^{\mathrm{a}}$ \\
\hline & $\operatorname{Tr}$ & $26.53 \pm 0.21^{\mathrm{e}}$ & $92.34 \pm 0.04^{\mathrm{abc}}$ & $7.66 \pm 0.07^{\mathrm{abc}}$ & $8.97 \pm 0.00^{e}$ & $94.03 \pm 0.00^{c}$ & $12.73 \pm 0.06^{\mathrm{ab}}$ & $2.22 \pm 0.16^{b c}$ & $6.23 \pm 0.01^{a}$ \\
\hline & $\mathrm{Mg}$ & $30.01 \pm 0.48^{\text {cd }}$ & $93.37 \pm 0.07^{\mathrm{bc}}$ & $9.63 \pm 0.57^{\mathrm{ab}}$ & $10.50 \pm 0.12^{\mathrm{bc}}$ & $89.50 \pm 0.12^{\text {ef }}$ & $8.45 \pm 0.46^{d}$ & $2.17 \pm 0.10^{b c}$ & $6.10 \pm 0.00^{\mathrm{a}}$ \\
\hline & $\mathrm{Fr}$ & $21.56 \pm 0.06^{f}$ & $91.75 \pm 0.57^{\mathrm{abc}}$ & $8.25 \pm 0.07^{\mathrm{abc}}$ & $6.54 \pm 0.11^{f}$ & $93.46 \pm 0.11^{b}$ & $8.20 \pm 0.41^{d}$ & $2.40 \pm 0.11^{b}$ & $5.89 \pm 0.04^{b}$ \\
\hline & $\mathrm{Ma}$ & $30.80 \pm 0.11^{b c}$ & $90.96 \pm 0.17^{\mathrm{abc}}$ & $9.04 \pm 0.17^{\mathrm{abc}}$ & $9.32 \pm 0.30^{\mathrm{de}}$ & $90.68 \pm 0.30^{\mathrm{cd}}$ & $13.94 \pm 0.15^{\mathrm{a}}$ & $2.50 \pm 0.12^{b}$ & $5.26 \pm 0.00^{d}$ \\
\hline \multirow{7}{*}{ Ma-PO7 } & $\mathrm{Mg}+\mathrm{Fr}$ & $31.67 \pm 0.74^{b}$ & $90.29 \pm 0.12^{\mathrm{bc}}$ & $9.71 \pm 0.12^{\mathrm{ab}}$ & $13.46 \pm 0.44^{\mathrm{a}}$ & $86.54 \pm 0.44^{9}$ & $9.95 \pm 0.76^{\mathrm{bcd}}$ & $3.09 \pm 0.18^{\mathrm{a}}$ & $5.37 \pm 0.17^{d}$ \\
\hline & $\mathrm{Mg}+\mathrm{Tr}$ & $28.89 \pm 0.54^{d}$ & $90.87 \pm 0.67^{\mathrm{abc}}$ & $9.13 \pm 0.17^{\mathrm{abc}}$ & $10.54 \pm 0.22^{\mathrm{bc}}$ & $89.46 \pm 0.22^{\mathrm{ef}}$ & $8.35 \pm 0.00^{d}$ & $2.21 \pm 0.02^{\mathrm{bc}}$ & $5.68 \pm 0.04^{c}$ \\
\hline & $\mathrm{Mg}+\mathrm{Ma}$ & $30.02 \pm 0.69^{c d}$ & $92.51 \pm 0.11^{\mathrm{abc}}$ & $7.49 \pm 0.11^{\mathrm{abc}}$ & $10.98 \pm 0.13^{b}$ & $89.02 \pm .0 .13^{f}$ & $9.21 \pm 0.40^{\mathrm{cd}}$ & $3.30 \pm 0.12^{\mathrm{a}}$ & $6.16 \pm 0.01^{a}$ \\
\hline & $\mathrm{Mg}+\mathrm{Fr}+\mathrm{Ma}$ & $37.78 \pm 0.36^{a}$ & $89.82 \pm 0.12^{c}$ & $10.18 \pm 0.12^{\mathrm{a}}$ & $11.04 \pm 0.71^{b}$ & $88.96 \pm 0.71^{f}$ & $11.75 \pm 0.37 \mathrm{abc}$ & $3.24 \pm 0.00^{\mathrm{a}}$ & $5.07 \pm 0.02^{\mathrm{e}}$ \\
\hline & $\mathrm{Mg}+\mathrm{Fr}+\mathrm{Tr}$ & $31.15 \pm 0.21^{b c}$ & $93.00 \pm 0.11^{\mathrm{ab}}$ & $7.00 \pm 0.11^{\mathrm{bc}}$ & $10.79 \pm 0.25^{b}$ & $89.21 \pm 0.25^{f}$ & $14.53 \pm 0.39^{a}$ & $3.15 \pm 0.03^{a}$ & $6.18 \pm 0.00^{\mathrm{a}}$ \\
\hline & $\mathrm{Mg}+\mathrm{Tr}+\mathrm{Ma}$ & $37.62 \pm 0.13^{a}$ & $90.01 \pm 0.19^{c}$ & $9.99 \pm 0.19^{a}$ & $9.83 \pm 0.09^{c d}$ & $90.17 \pm 0.09 \mathrm{de}$ & $9.93 \pm 0.44^{\mathrm{bcd}}$ & $2.50 \pm 0.32^{\mathrm{b}}$ & $5.08 \pm 0.01^{e}$ \\
\hline & $P$ & 0.001 & 0.0075 & 0.0075 & 0.012 & 0.012 & 0.022 & 0.0001 & 0.001 \\
\hline
\end{tabular}

Proteína cruda (PC); humedad (H); materia seca (MS); ceniza (Ce); materia orgánica (MO); fibra cruda (FC); extracto etéreo (EE). Tr= Paja de trigo; $F r=$ Paja de frijol; Ma= Rastrojo de maíz; Mg= Penca de maguey; Mg+Tr= Penca de maguey + paja de trigo (1:1); Mg+Ma= Penca de maguey + rastrojo de maíz (1:1); Mg+F= Penca de maguey + paja de frijol (1:1); Mg+Tr+Fr= Penca de maguey + paja de trigo + paja de frijol (1:1:1); Mg+Ma+Tr= Penca de maguey + rastrojo de maíz + paja de trigo (1:1:1); Mg+Ma+Fr= Penca de maguey + rastrojo de maíz + paja de frijol (1:1:1).

*Medias con letras diferentes indican diferencias significativas con la prueba de Tukey $(P \leq 0.05)$.

$\mathrm{P}=$ Significancia. 
tíferos del $P$. opuntiae presentó diferencias significativas entre los tratamientos, oscilando entre 7 y 10.18 $\%$, mientras que los basidiomas obtenidos de la cepa CP-50 fue de $6.72 \%$. Estos valores están dentro del intervalo obtenido en otros estudios, así Alam et al. (2008) reportaron $14 \%$ en $P$. ostreatus, $13 \%$ en $P$. sajor-caju y 12.5 a $14 \%$ en $P$. florida. Sin embargo, estudios recientes como el de Oyetayo y Ariyo (2013), reportaron $9.25,10.72$ y $2.22 \%$ con $P$. ostreatus cultivado en sustratos de madera de Pycnanthus ongoleubis, Ceiba pentandra y Cananium sp, respectivamente. Así mismo, Ogundele et al. (2014) encontraron 9.34 \% en basidiomas cultivados con aserrín de Daniellia oliveri y salvado de arroz, inoculados con P. ostreatus. El porcentaje de cenizas (Ce) obtenido de los cuerpos fructíferos de la cepa Ma-PO7 presentó deferencias estadísticas significativas en la prueba de Tukey $(P=0.012)$, con valores entre $2.45 \pm 0.12$ hasta $11.04 \pm 0.71 \%$. El resultado fue bastante similar a los hallazgos de Hoa et al. (2015) quienes reportaron un contenido de cenizas del 5.90 a $7.10 \%$ para $P$. ostreatus cultivado en una mezcla de aserrín y bagazo de caña (80:20); estos resultados son superiores a los encontrados en la CP-50 (2.45 \%) de la presente investigación.

Los porcentajes de materia orgánica (MO) en los cuerpos fructíferos de P. opuntiae osciló entre 86.54 y $94.03 \%$, sin embargo, la CP-50 obtuvo $97.55 \%$ en el tratamiento control (Tabla 3). Estudios de Yamauchi et al. (2018) reportaron $92.9 \%$ de MO en basidiomas cultivados en aserrín de bambú fermentado y salvado de arroz (1:1), resultados similares en el presente estudio. Ahmed et al. (2013) reportaron $88.5 \%$ de MO en $P$. highking y $88.6 \%$ en $P$. ostreatus, cultivados en aserrín suplementado con salvado de trigo. Así mismo, Finimundy et al. (2018) reportan $94.6 \%$ de $\mathrm{MO}$ en basidiomas de $P$. sajor caju, resultados similares a los encontrados en la cepa Ma-PO7 de P. opuntiae. En cuanto a la fibra cruda (FC), los basidiomas de $P$. opuntiae presentaron un porcentaje promedio de $10.34 \%$ y la cepa de P. ostreatus $14.67 \%$, en el grupo control. Oyetayo y Ariyo (2013) reportaron 17.51 \% de FC en carpóforos de $P$. ostreatus, cultivados en madera de Pycnanthus ongoleubis; mientras que Hoa et al. (2015) reportaron $29.75 \%$ para $P$. ostreatus, usando como sustrato olote de maíz, resultados mayores a los reportados en la presente investigación. En cuanto al extracto etéreo (EE) contenido en los basidiocarpos de P. opuntiae, el tratamiento $\mathrm{Mg}+\mathrm{Ma}$ presentó el mayor contenido con
$3.30 \%$ y el grupo control alcanzó el porcentaje más bajo, $1.95 \%$; cabe mencionar que los tratamientos con la cepa Ma-PO7 mostraron más contenido de grasa que la cepa CP-50. Hoa et al. (2015) reporta para $P$. ostreatus un $2.78 \%$ de grasas totales en una mezcla 80:20 de aserrín y bagazo de caña.

\section{CONCLUSIONES}

La cepa comercial CP-50 de P. ostreatus alcanzó la mayor producción total, eficiencia biológica y la tasa de producción en comparación con la cepa Ma-PO7 de P. opuntiae. Sin embargo, los basidiomas de la cepa Ma-PO7 presentaron los porcentajes de proteína cruda, materia seca, cenizas y extracto etéreo más altos, lo que le da un valor nutrimental mayor que la cepa comercial de P. ostreatus. CP-50.

La combinación de los sustratos agrícolas con la penca de maguey favoreció un mayor rendimiento de basidiomas de la cepa de P. opuntiae, así como un incremento en el contenido de humedad y materia orgánica.

Los resultados obtenidos con la cepa Ma-PO7 son prometedores y abren la posibilidad de cultivar esta especie a nivel piloto en la región de Tetela de Ocampo, Puebla (México) debido a que ya existe un consumo basado en el conocimiento tradicional de esta especie y en la producción comercial de otras especies del género Pleurotus. Esto permitiría, además de aprovechar los residuos agrícolas locales, impulsar el desarrollo tecnológico en la producción de setas de maguey en la sierra norte del estado de Puebla.

\section{AGRADECIMIENTOS}

Los autores agradecen a la Vicerrectora de Investigación y Estudios de Posgrado de la Benemérita Universidad Autónoma de Puebla (BUAP) y al CONACyT por el financiamiento otorgado a este proyecto de investigación con CVU: 775054.

\section{LITERATURA CITADA}

AOAC, 1990. Association of Official Analytical Chemists: Direct method. Numbers 920.34, 934.01, 942.05. 16a Edit. Ed. Association of Official Analytical Chemists, International Gaithersburg.

AOAC, 2004. Official Methods of Analysis International. 19th ed., Gaithersburg, Md.: AOAC International, ISBN: 978-0-93558483-7.

Ahmed, M., N. Abdullah, U. Ahmed, B.M. Kamal, H.M. Bhuyan, 2013. Yield and nutritional composition of oyster mushroom strains newly introduced in Bangladesh. Pesquisa Agropecuaria Brasileira 48(2): 197-202. 
Alam, N., R. Amin, A. Khan, I. Ara, M.J. Shim, M.W. Lee, T.S. Lee, 2008. Nutritional analysis of cultivated mushrooms in Bangladesh: Pleurotus ostreatus, Pleurotus sajor-caju, Pleurotus florida and Calocybe indica. Mycobiology 36(4): 228-232.

Andrino, A., A. Morte, M. Honrubia, 2011. Caracterización y cultivo de tres cepas de Pleurotus eryngii (Fries) Quélet sobre sustratos basados en residuos agroalimentarios. Anales de Biología 33: 53-66.

$A R C, 1980$. The nutrient requeriments of ruminant livestock. CAB, Londres.

Bandopadhyay, S., 2013. Effect of supplementing rice straw with water hyacinth on the yield and nutritional qualities of Oyster mushrooms (Pleurotus spp.). Micología Aplicada International 25(2): 15-21

Barrales, M., G. Mata, 2016. Selección de cepas del hongo de maguey (Pleurotus opuntiae) y evaluación de su producción en sustratos fermentados. Interciencia 41(5): 346-352.

Barros, L., T. Cruz, P. Baptista, L.M. Estevinho, C.R. Ferreira, 2008. Wild and commercial mushrooms as source of nutrients and nutraceuticals. Food and Chemical Toxicology 46: 2742-2747.

Bederska-Lojewska, D., S. Świątkiewicz, B. Muszyńska, 2017. The use of Basidiomycota mushrooms in poultry nutrition: a review. Animal Feed Science and Technology 230: 1-40.

Camacho, G., G. Guzmán, L. Guzmán-Dávalos, 2012. Pleurotus opuntiae (Durieu et Lev.) Sacc. (Higher Basidiomycetes) and other species related to Agave and Opuntia plants in Mexico: Taxonomy, distribution and applications. International Journal of Medicinal Mushrooms 14(1): 65-78.

Çağlarırmak, N., 2007. The nutrients of exotic mushrooms (Lentinula edodes and Pleurotus species) and an estimated approach to the volatile compounds. Food Chemistry 105: 1188-1194.

Cayetano-Catarino, M., T. Bernabé-González, 2008. Cultivo de Pleurotus sobre residuos de las cosechas de jamaica (Hibiscus sabdariffa) y plátano (Musa paradisiaca). Revista Mexicana de Micología 26: 57-60.

Carrasco-González, J.A., S.O. Serna-Saldívar, J.A. Gutiérrez-Uribe, 2017. Nutritional composition and nutraceutical properties of the Pleurotus fruiting bodies: Potential use as food ingredient. Journal of Food Composition and Analysis 58: 39-81.

Carvalho, de C.S.M., C. Sales-Campos, L.P. de Carvalho, M.T. de Almeida Minhoni, A.L.M. Saad, G.P. Alquati, M.C.N Andrade, 2015. Cultivation and bromatological analysis of the medicinal mushroom Ganoderma lucidum (Curt.: Fr.) P. Karst cultivated in agricultural waste. African Journal of Biotechnology 14(5): 412-418.

Chairez-Aquino, J., R.J. Enríquez-del-Valles, J. Ruíz-Luna, G.V. Campos-Ángeles, R. Martínez-García, 2015. Uso del bagazo de Agave spp. y hojas de maíz para cultivar el hongo Pleurotus ostreatus. Revista Mexicana de Agroecosistemas 2(1): 23-28.

Das, C., E. López de León, L.F. Pascual, M. Battaglia, 2010. Guía técnica de producción de hongos comestibles de la especie Pleurotus ostreatus. Journal of Agriculture and Environment for International Development 104 (3-4): 139-154.

Feldsine, P., C. Abeyta, W.H. Andrews, 2002. AOAC International methods committee guidelines for validation of qualitative and quantitative food microbiological official methods of analysis. Journal of AOAC International 85(5): 1187-200.
Finimundy, C.T., L. Barros, C.R. Calhelha, J.M. Alves, A.M. Prieto, V.M.R. Abreu, P.J.A. Dillon, P.A.J. Henriques, M. Roesch-Ely, R.F.C.I. Ferreira, 2018. Multifunctions of Pleurotus sajor-caju (Fr.) Singer: A highly nutritious food and a source for bioactive compounds. Food Chemistry 245: 150-158.

García-Oduardo, N., S.R. Bermúdez, M. Serrano-Alberni, 2011. Formulaciones de sustratos en la producción de setas comestibles Pleurotus. Tecnología Química (3): 15-22.

Garzón, G.J., A.J. Cuervo, 2008. Producción de Pleurotus ostreatus sobre residuos sólidos lignocelulósicos de diferente procedencia. Ciencias Biomédicas 6(10): 126-140.

Gomes, C.R.C., T. Brugnari, A. Bracht, P.R. Marina, C.F.R. I. Ferreira, 2016. Biotechnological, nutritional and therapeutic uses of Pleurotus spp. (Oyster mushroom) related with its chemical composition: A review on the past decade findings. Trends in Food Science and Technology 50: 103-117.

Girmay, Z., W. Gorems, G. Birhanu, S. Zewdie, 2016. Growth and yield performance of Pleurotus ostreatus (Jacq. Fr.) Kumm (oyster mushroom) on different substrates. AMB Express 6: 87.

Heredia-Solis, A., E. Esparza-lbarra, L. Romero-Bautista, F. Cabral-Arellano, R. Bañuelos-Valenzuela, 2014. Bagazos de Agave salmiana y Agave weberi utilizados como sustrato para producir Pleurotus ostreatus. Revista Iberoamericana de Ciencias 1(5): 103-110.

Hoa, H.T., C.L. Wang, C.-H. Wang, 2015. The effects of different substrates on the growth, yield, and nutritional composition of two oyster mushrooms (Pleurotus ostreatus and Pleurotus cystidiosus). Mycobiology 43(4): 423-434.

Keles, A., I. Koca, H. Gençcelep, 2011. Antioxidant properties of wild edible mushrooms. Journal of Food Process and Technology 2(6): 130-143.

Kurt, S., S. Buyukalaca, 2010. Yield performances and changes in enzyme activities of Pleurotus spp. (P. ostreatus and P. sajor-caju) cultivated on different agricultural wastes. Bioresource Technology 101: 3164-3169.

López, A.V.C., V.N Martínez, S.M. Basurto, L.R. Pérez, 2010. Los compuestos esteroidales o saponinas en la palma del desierto (Yucca schidigera) y sus aplicaciones. Aventuras del Pensamiento 3 : 16-17.

Michael H.W., G. Bultosa, L.M. Pant, 2011. Nutritional contents of three edible oyster mushrooms grown on two substrates at Haramaya, Ethiopia, and sensory properties of boiled mushroom and mushroom sauce. International Journal of Food Science and Technology 46: 732-738.

Ogundele, G.F., R.O. Abdulazeez, O.P. Bamidele, 2014. Effect of pure and mixed substrate on oyster mushroom (Pleurotus ostreatus) cultivation. Journal of Experimental Biology and Agricultural Sciences 2(2S): 2320-8694.

Oyetayo, V.O., O.O. Ariyo, 2013. Micro and macronutrient properties of Pleurotus ostreatus (Jacq: Fries) cultivated on different wood substrates. Jordan Journal of Biological Sciences 6(3): 223-226.

Patel, Y., R. Naraian, V.K. Singh, 2012. Medicinal properties of Pleurotus species (Oyster mushroom): A Review. World Journal of Fungal and Plant Biology 3(1): 1-12.

Romero, O., M.M.A. Huerta, A. Damián, A. Macías, M.A. Tapia, J.F. Parraguirre, J. Juárez, 2010. Evaluación de la capacidad produc- 
tiva de Pleurotus ostreatus con el uso de hoja de plátano (Musa paradisiaca L., cv. Roatan) deshidratada, en relación con otros sustratos agrícolas. Agronomía Costarricense 34(1): 53-63.

Rui-Heng, Y., L. Yan, W. Ying, W. Jia-Ning, Z. Chen-Li, W. Ying, G. Ying-Nv, M. Wen-Jun, T. Li-Hua, G. Ming, W. Ying-Ying, B. DaPeng, 2016. The genome of Pleurotus eryngii provides insights into the mechanisms of wood decay. Journal of Biotechnology 239: 65-67.

Salmones D., R. Gaitán-Hernández, R. Pérez, G. Guzmán, 1997. Estudios sobre el género Pleurotus. Interacción entre crecimiento micelial y productividad. Revista Iberoamericana de Micología, 14: $173-176$.

Sánchez, C., 2010. Cultivation of Pleurotus ostreatus and other edible mushrooms. Applied Microbiology and Biotechnology 85: 1321-1337.

Seibel, W., 1989. Approved methods of the American Association of Cereal Chemists. 8th ed. Starch. Paul, Minnesota.
Sobal, M., D. Martínez-Carrera, P. Morales, S. Roussos, 2007. Classical characterization of mushroom genetic resources from temperate and tropical regions of México. Micología Aplicada International 19(1): 15-23.

Tolera, K.D, S. Abera, 2017. Calidad nutricional del hongo oyster (Pleurotus ostreatus) según se ve afectado por los pretratamientos osmóticos y los métodos de secado. Food Science and Nutrition 5(5): 989-996.

Thongklang, N., T. Luangharn, 2016. Testing agricultural wastes for the production of Pleurotus ostreatus. Mycosphere 7(6): 766772.

Van Soest, P.J. 2002. Nutritional Ecology of the ruminant, second ed. Comstock Publishing Associates. Cornell University Press, Ithaca.

Yamauchi, M., M. Sakamoto, M. Yamada, H. Hara, T.S. Mat, S. Rezania, D.M. Fadhil, H.F. Mohd, 2018. Cultivation of oyster mushroom (Pleurotus ostrreatus) on fermented moso bamboo sawdust. Journal of King Saud University Science (en prensa). 